\title{
Comparison between endonasal endoscopic polyp size scores and quality of life outcome after optimal medical treatment*
}

\author{
Saleh Khaled Aboud', Salina Husain², Balwant Singh Gendeh² \\ ' Department of Otorhinolaryngology - Head and Neck Surgery, Malaysian Allied Health Sciences Academy (MAHSA) University, \\ Jalan Elmu off Jalan Universiti, Kuala Lumpur, Malaysia \\ 2Department of Otorhinolaryngology, Universiti Kebangsaan Malaysia, Cheras, Kuala Lumpur, Malaysia
}

Rhinology 52: 334-340, 2014

DOl:10.4193/Rhino14.029

*Received for publication:

February 28, 2014

Accepted: April 13, 2014

\begin{abstract}
Background: The aim of this prospective study is to evaluate whether changes in endoscopy score correlate with changes in quality of life (QOL) in nasal polyposis (NP) patients after optimal medical therapy.

Methodology: An interventional clinical trial study involving fifty five patients with grade I and II NP was conducted. Patients were initially treated with a single dose oral prednisolone $25 \mathrm{mg}$ for 2 weeks, Macrolide $250 \mathrm{mg}$ daily for the first 3 months and long-term intranasal steroids. All patients were followed up and evaluated subjectively by Rhinosinusitis Disability Index questionnaire and objectively by endonasal endoscopy between December 2011 to October 2013.
\end{abstract}

Results: At baseline, patients showed significantly bad QOL scores on all domains. At 3 months, a significant improvement in all impaired QOL domains and reduction of the nasal polyp size were demonstrated compared to baseline. At 6 and 12 months, no significant improvement between QOL scores and nasal polyp size was observed.

Conclusion: Optimal medical treatments improve both the QOL and nasal polyp size in grade I and II NP patients. However, the changes in endoscopy scores explain a part of the improvement in QOL outcomes as the latter are multidimensional constructs.

Key words: nasal polyposis, quality of life, medical polypectomy, endonasal endoscopy

\section{Introduction}

Nasal polyposis is characterized by a recurrent sinonasal mucosal inflammatory process with presence of two or more symptoms of obstruction, congestion or discharge, with or without facial pain/pressure or reduced sense of smell for more than 12 weeks with pathologic endoscopic findings and/or CT scan changes within the ostiomeatal complex and/or sinuses

(1). Nasal endoscopy has a definite role in detecting nasal polyps (2) The aetiology of NP is unknown. The prevalence of NP in the general population is considered to be around $4 \%{ }^{(3)}$ and can be associated with different systemic and respiratory diseases such as cystic fibrosis, rhinitis, and asthma with or without aspirin sensitivity. Approximately $4.6 \%$ have acetylsalicylic acid-triad: bronchial asthma, chronic rhinosinusitis and aspirin insensitivity (4). Patients with NP seem to have a more pronounced disease burden than those without polyps ${ }^{(5)}$. NP is primarily managed medically and optimal medical therapy is needed before and after surgery. A short course systemic steroid (25 mg prednisolone daily for 14 days) serving as "Medical polypectomy" is equally effective as simple polypectomy with a snare ${ }^{(6)}$.

Intranasal corticosteroid as basic long-term therapy clearly reduces the size of nasal polyps with a significant effect on blockage symptom scores, but has little effect on the loss of the sense of smell ${ }^{(7)}$. Macrolide antibiotics have high anti-inflammatory activities, decrease virulence of colonizing bacteria and cause shrinkage of nasal polyps by suppressing cytokine production by inflammatory cells in the paranasal sinus epithelium ${ }^{\left({ }^{8}\right)}$. Topical steroids have been investigated extensively and compared in 298 subjects with moderate NP where mometasone furoate nasal spray or placebo was given for 16 weeks. A significant decrease in nasal congestion, polyp size, and improved quality of life 
in the momentasone furoate - treated patients was observed ${ }^{(9)}$. Similar findings were reported after treatment of 20 NP patients with chronic sinusitis with $150 \mathrm{mg}$ roxithromycin once a day for 8 weeks. After completion of the macrolide treatment, a reduction in the size of nasal polyps was observed in more than half of the treated patients ${ }^{(10)}$. Sinonasal endoscopy is used to gather critical information about mucosal inflammation before and after therapeutic intervention. QOL is the result of interactions among many factors and its improvement is a complex process that cannot be explained only by improved endoscopic findings. Accordingly, it is not surprising that the QOL does not always correlate with results of other objective tests. The aim of this study was to investigate the relationship between change in endoscopy score and change in QOL following optimal medical therapy. We hypothesized that improvement in endoscopy scores significantly correlates with improvement in QOL following optimal medical treatment.

\section{Materials and methods}

Study population

A total of 55 Patients with NP grades I and II using the Lildholdt classification were included in this prospective study ${ }^{(11)}$. The duration of the study was from April 2011 to February 2013 with age range of 18 to 65 years with mean age of 51.47 (SD \pm 14.35 ). Females outnumbered males (54.5\% and $45.5 \%$ ). All patients were examined and treated for the entire duration of the study at the outpatient Otorhinolaryngology clinic. Approval for this study was obtained from the Ethics Committee/IRB, REF NO: UKM 1.5.3.5/244/FF-121-2011 and a signed informed consent was obtained from all patients.

\section{Inclusion and exclusion criteria}

The inclusion criteria were patient age between $18-65$ years, nasal symptoms more than 12 week and NP grades I and II, while the exclusion criteria were NP grade III (high indication for surgical treatment), surgically treated patient with nasal polyposis, allergy to macrolide antibiotics, patients with absolute contraindications for systemic steroids and patients with severe structural abnormalities.

\section{Study design}

After a 4-week washout period of both oral and intranasal steroids (week 0), 55 patients received systemic steroid (oral prednisone) $25 \mathrm{mg}$ in single dose for 2 weeks, followed by maintenance long-term intranasal steroid (e.g. mometasone furoate aqueous nasal spray $200 \mathrm{mcg} /$ day). Macrolide (Clarithromycin) antibiotic $250 \mathrm{mg}$ daily was given as single dose for the first 3 months. An initial baseline evaluation before treatment was performed followed by three follow-up evaluations after 3 months, 6 months and 12 months of treatment. QOL were scored by Rhinosinusitis Disability Index (RSDI) questionnaire.
All the recruited patients had sinonasal endoscopy performed at 3,6 , and 12 months after start of the treatment.

\section{Quality of life and Rhinosinusitis Disability Index assess- ment} All patients with grade I and II NP fulfilling the inclusion criteria were assessed subjectively before and after medical treatment by filling the RSDI Questionnaire comprising of 28 questions divided into three domains (physical, mental and social). Scale scores range from 0 to $100 \%$ and lower scores indicate better QOL. Each domain has ten levels of answers based on tables, which permit only one answer. These scores were taken and compared at the different follow-up study interval (baseline, 3 months, 6 months and a year).

\section{Polyp size score}

Using rigid endoscopy, polyp size was scored from 0 to 3 for each nasal cavity (Lildholdt classification): 0 , no polyps; 1 , mild polyposis (small polyps not reaching the upper edge of the inferior turbinate); 2 , moderate polyposis (polyps between the upper and lower edges of the inferior turbinate); 3 , severe polyposis (large polyps reaching the lower edge of the inferior turbinate).

\section{Statistics}

Data analysis was performed with the statistical package IBM SPSS version 19.0 using mean \pm SD (standard deviation). A pvalue $<0.05$ was considered statistically significant. All data was assessed for normal distribution. ANOVA was used to compare 55 patients' percentages of QOL and nasal polyp size scores at varying unit times in the same population.

\section{Results}

A total number of 55 NP patients were prospectively recruited and enrolled into the study with baseline comparison of disease-severity scores (QOL scores) with nasal polyp size scores over one year follow-up. All patients received medical co-interventions, which include oral prednisolone, Clarithromycin and continuous intranasal steroid application and allergy therapy. Follow-up data was available for 55 (91\%) of the 60 patients at the end of this study.

\section{Frequency of various diseases in nasal polyps}

Prior to treatment, twenty patients with NP were diagnosed as hypertensive (36.4\%). Allergic rhinitis and asthmatic patients were seen in 17 (30.9\%) and 19 (34.5\%) of NP patients, respectively. Eczematous disease was reported in $5(9.1 \%)$ patients and aspirin intolerance in only 3(5.5\%). Amongst 55 patients with nasal polyposis only 39 (70.9\%) were under medication (Table $1)$. 
Table 1. Number and percentages of disease condition by duration visit $(n=55)$.

\begin{tabular}{|c|c|c|c|c|c|c|c|c|}
\hline \multirow[b]{2}{*}{ Disease } & \multicolumn{2}{|c|}{ Baseline } & \multicolumn{2}{|c|}{3 Month } & \multicolumn{2}{|c|}{6 Month } & \multicolumn{2}{|c|}{1 year } \\
\hline & $\mathbf{N}$ & $\%$ & $\mathbf{N}$ & $\%$ & $\mathbf{N}$ & $\%$ & $\mathbf{N}$ & $\%$ \\
\hline DM & 12 & 21.8 & 9 & 16.4 & 7 & 12.7 & 8 & 14.5 \\
\hline HT & 20 & 36.4 & 18 & 32.7 & 17 & 30.9 & 17 & 30.9 \\
\hline Asthma & 19 & 34.5 & 16 & 29.1 & 15 & 27.3 & 16 & 29.1 \\
\hline AR & 17 & 30.9 & 16 & 29.1 & 15 & 25.5 & 15 & 17.3 \\
\hline Osteoporosis & 1 & 1.8 & 1 & 1.8 & 1 & 1.8 & 1 & 1.8 \\
\hline Aspirin intolerance & 3 & 5.5 & 3 & 5.5 & 3 & 5.5 & 3 & 5.5 \\
\hline Eczema & 5 & 9.1 & 5 & 9.1 & 5 & 9.1 & 5 & 9.1 \\
\hline Hypothyroidism & 2 & 3.6 & 2 & 3.6 & 2 & 3.6 & 2 & 3.6 \\
\hline
\end{tabular}

$\mathrm{DM}=$ diabetes mellitus; $\mathrm{HT}$ = hypertension; $\mathrm{AR}=$ allergic rhinitis.

\section{Quality of life (RSDI) assessment}

ANOVA was used to compare 55 patients' percentages of QOL for different duration visits. Pair wise comparisons showed the QOL baseline $(40.52 \pm 12.07)$ was significantly more at 3 month (23.66 \pm 9.85$), 6$ month $(18.32 \pm 8.42)$ and 1 year $(17.28 \pm 8.79)$ follow-up. All patients with NP had significantly worse QOL scores before treatment. At 3 months, 6 months and 12 months, patients with NP showed a similar significant improvement in QOL. No significant differences were observed on QOL between 6 months and 12 months. In all domains of RSDI, patients with NP showed significantly worse physical function $(4.59 \pm 1.41)$ than social function ( $3.16 \pm 1.17$ ) of QOL scores. All the RSDI domains are statistically correlated to NP patients with asthma (Table 2). At baseline, asthmatic patients with NP had worse scores of QOL $(p<0.05)$ than non-asthmatic with NP in physical, mental and social functioning. Before treatment asthmatic patients showed lower physical, mental and social functioning than non-asthmatic patients. At 6 and 12 months, similar QOL improvement were observed for physical and mental functions in asthmatic and non-asthmatic patients except for social function where the former had worse QOL score than the latter.

\section{Nasal polyps size score}

Before treatment, no significant differences were found in the polyp size between the right and left nasal cavity. At 3 months, medical treatment caused a significant reduction of the polyp size when compared with baseline $(p<0.05)$. No significant differences in polyp size either between asthmatic and non-asthmatic patients wer observed before treatment and at 3 months, 6 months and 12 months (Table 3 ).

A significant difference in polyp size score between right and
Table 2. Summary of comparison of mean of physical, mental and social components and mean difference in asthmatic and non-asthmatic patients with nasal polyposis.

\begin{tabular}{|c|c|c|c|c|}
\hline \multicolumn{5}{|c|}{ Physical } \\
\hline & $\begin{array}{c}\text { All } \\
(n=55)\end{array}$ & $\begin{array}{l}\text { No asthma } \\
\quad(n=33)\end{array}$ & $\begin{array}{l}\text { Asthma } \\
(n=19)\end{array}$ & $\begin{array}{c}\text { Mean } \\
\text { difference }\end{array}$ \\
\hline T0 & $4.59 \pm 1.41$ & $4.48 \pm 1.43$ & $4.79 \pm 1.37$ & 0.31 \\
\hline T3 & $2.80 \pm 1.27^{*}$ & $2.65 \pm 1.23^{*}$ & $3.09 \pm 1.32^{*}$ & 0.44 \\
\hline T6 & $2.21 \pm 0.99 *$ & $2.18 \pm 1.08^{*}$ & $2.27 \pm 0.82^{*}$ & 0.09 \\
\hline T12 & $2.03 \pm 1.05^{*}$ & $2.04 \pm 1.17^{*}$ & $2.02 \pm 0.81^{*}$ & 0.02 \\
\hline \multicolumn{5}{|c|}{ Mental } \\
\hline & $\begin{array}{c}\text { All } \\
(n=55)\end{array}$ & $\begin{array}{l}\text { No asthma } \\
(n=33)\end{array}$ & $\begin{array}{l}\text { Asthma } \\
(n=19)\end{array}$ & $\begin{array}{c}\text { Mean } \\
\text { difference }\end{array}$ \\
\hline T0 & $3.70 \pm 1.19$ & $3.58 \pm 1.10$ & $3.92 \pm 1.34$ & 0.34 \\
\hline T3 & $2.07 \pm 0.83^{*}$ & $2.00 \pm 0.89^{*}$ & $2.20 \pm 0.69^{*}$ & 0.20 \\
\hline T6 & $1.59 \pm 0.81^{*}$ & $1.56 \pm 0.86^{*}$ & $1.65 \pm 0.73^{*}$ & 0.09 \\
\hline T12 & $1.49 \pm 0.95^{*}$ & $1.50 \pm 1.01^{*}$ & $1.48 \pm 0.86^{*}$ & 0.02 \\
\hline \multicolumn{5}{|c|}{ Social } \\
\hline & $\begin{array}{c}\text { All } \\
(n=55)\end{array}$ & $\begin{array}{l}\text { No asthma } \\
\quad(n=33)\end{array}$ & $\begin{array}{l}\text { Asthma } \\
(n=19)\end{array}$ & $\begin{array}{c}\text { Mean } \\
\text { difference }\end{array}$ \\
\hline T0 & $3.16 \pm 1.17$ & $3.05 \pm 1.15$ & $3.36 \pm 1.22$ & 0.31 \\
\hline T3 & $1.65 \pm 0.81^{*}$ & $1.60 \pm 0.85^{*}$ & $1.77 \pm 0.75^{*}$ & 0.17 \\
\hline T6 & $1.53 \pm 1.42^{*}$ & $1.33 \pm 0.73^{*}$ & $1.91 \pm 2.19^{*}$ & 0.58 \\
\hline T12 & $1.24 \pm 0.77^{*}$ & $1.21 \pm 0.69^{*}$ & $1.30 \pm 0.91 *$ & 0.09 \\
\hline
\end{tabular}

T0, Baseline; T3, 3 Months; T6, 6 Months; T12, 1 Year, *Paired t test with baseline, $\mathrm{p}<0.001$. 
Table 3. Polyp size scores at baseline, 3, 6 and 12 months of treatment.

\begin{tabular}{|c|c|c|c|c|c|c|}
\hline Asthma & Time & $\mathbf{n}$ & Mean polyp size & SD & $\mathbf{t}$ & $\mathbf{p}$ \\
\hline \multirow[t]{6}{*}{ No } & TNCTO & 72 & 1.50 & 0.50 & 5.96 & $0.001^{* *}$ \\
\hline & TNCT3 & 72 & 0.90 & 0.87 & & \\
\hline & TNCTO & 72 & 1.50 & 0.50 & 6.30 & $0.001^{* *}$ \\
\hline & TNCT6 & 72 & 0.86 & 0.70 & & \\
\hline & TNCTO & 72 & 1.50 & 0.50 & 6.37 & $0.001^{* *}$ \\
\hline & TNC T12 & 72 & 0.83 & 0.71 & & \\
\hline \multirow[t]{6}{*}{ Yes } & TNCTO & 36 & 1.50 & 0.51 & 2.23 & $0.03^{* *}$ \\
\hline & TNCT3 & 36 & 1.18 & 0.69 & & \\
\hline & TNCTO & 36 & 1.50 & 0.51 & 3.41 & $0.001^{* *}$ \\
\hline & TNCT6 & 36 & 0.97 & 0.79 & & \\
\hline & TNCTO & 36 & 1.50 & 0.51 & 4.13 & $0.001^{* *}$ \\
\hline & TNC 12 & 36 & 0.87 & 0.81 & & \\
\hline
\end{tabular}

TNC $=$ Total Nasal Cavity, $\mathrm{T} 0=$ Baseline, $\mathrm{T} 3=3$ months, $\mathrm{T} 6=6$ months, $\mathrm{T} 12=1$ year. *Paired $\mathrm{t}$ test with baseline, $\mathrm{p}<0.05$

Table 4. Correlation between quality of life by time and right and left nasal cavity with nasal polyposis.

\begin{tabular}{|c|c|c|c|c|c|c|c|c|}
\hline & \multicolumn{2}{|c|}{ QOL 1} & \multicolumn{2}{|c|}{ QOL 2} & \multicolumn{2}{|c|}{ QOL 3} & \multicolumn{2}{|c|}{ QOL 4} \\
\hline & $r$ & $\mathbf{P}$ & $r$ & $\mathbf{P}$ & $r$ & $P$ & $\mathbf{r}$ & $\mathbf{P}$ \\
\hline RNC & 0.49 & $0.001^{* *}$ & 0.58 & $0.001^{* *}$ & 0.63 & $0.001^{* *}$ & 0.62 & $0.001^{* *}$ \\
\hline LNC & 0.52 & $0.001^{* *}$ & 0.68 & $0.001^{* *}$ & 0.64 & $0.001^{* *}$ & 0.69 & $0.001^{* *}$ \\
\hline
\end{tabular}

RNC = Right nasal cavity, LNC = Left nasal cavity, QOL1 = quality of life at baseline, QOL2 = quality of life at 3 months, QOL $3=$ quality of life at 6 months, QOL4 $=$ quality of life at 1 year post optimal medical treatment. $r=$ Correlation, ${ }^{* *}$ Significance $p<0.05$.

left nasal cavity was observed in aspirin tolerant (AT) patients before and after short course of oral prednisolone and longterm intranasal steroid, while no significant difference was noted in polyp size between right and left nasal cavities in aspirin intolerant patients $(n=3)$.

\section{Comparison between QOL and nasal polyposis from base-} line till 12 months follow-up

Complete data were obtained from 55 patients with nasal polyposis comparing nasal polyp size with QOL scores. Each subjective and objective parameter showed significant improvement in its value after medical treatment in comparison to before the treatment.

There was a significantly positive percentage of improvement in the polyp size score and nasal symptoms particularly nasal obstruction and loss of sense of smell in all RSDI domains (physical, mental, social) at 3 months. At 6 months no significant comparison in the nasal obstruction and polyp size score was determined $(p<0.05)$. The statistical scoring on QOL showed a positive correlation (Pearson) that was significant for the left nasal cavity particularly for QOL4 $(r=0.69, p=0.001)$. The correlation (Pearson) for the right nasal cavity was also positive but its significance is moderately lower than the left nasal cavity for QOL4 ( $r=0.49, \mathrm{p}=0.001$ ) (Table 4).

The main statistical methods that have been used are the t-test to compare two mean different groups, the paired-t-test to compare two means of different groups at different times, one way repeated measures ANOVA and Pearson correlation. 


\section{Discussion}

The management of NP should be primarily based on a medical approach, only complemented by surgical procedures in the case of drug failure. The changes in the management of adults with CRS with NP are subtle and the treatment should be based on the severity of symptoms using a Visual Analogue Score and endoscope ${ }^{(12)}$. This study has been developed to highlight the effects of treatment of NP on QOL and nasal polyp size after optimal medical treatment. All patients with polyposis score of 1 or 2 by the Lildholdt classification were treated with the same regime ${ }^{(13)}$. Our study highlighted that (i) patients with NP have a significantly worse QOL in all RSDI domains, where physical functioning was lower than the mental functioning) (ii) steroid treatment lead to similar improvement in all QOL domains in NP with or without asthma except for social functioning where asthmatic patients had worse QOL score than non-asthmatic (iii) both steroid and Clarithromycin treatments maintain improvement in nasal symptoms and (iv) Significant improvement in endoscopy score correlates with significant improvement in disease-specific QOL domains for patients managed with optimal medical therapy. Nasal symptoms clearly improved after oral steroid treatment and intranasal steroids maintained this effect, except for sense of the smell, which was more a drawback at 12 months in non-asthmatic than in asthmatic patients. There was evidence of a clear improvement in nasal symptoms after two weeks of oral steroids especially for nasal obstruction and reduced sense of smell ${ }^{(14)}$. Intranasal steroids (fluticasone or beclomethasone) have gained acceptance as an alternative treatment to surgery for NP and are effective in reducing nasal symptoms when compared to placebo ${ }^{(15)}$. Combined oral and intranasal steroids seem to be effective to treat NP by improving the sense of smell, nasal obstruction, and other nasal symptoms (16). Nasal obstruction is a major complaint in NP patients and the long lasting correction of olfactory dysfunction can be achieved through the combination of nasalization and low dose of nasal steroids ${ }^{(17)}$. Three major studies were dedicated to systemic steroid efficacy, which show a genuine improvement on all symptoms, especially on anosmia, which correlates well with the polyp's volume reduction ${ }^{(5,7,14)}$. Short-term systemic steroid administration thus appears to have the same efficacy as polypectomy, but the improvement proves to be of short duration ${ }^{(7)}$. In a Cochrane review, only one out of 17 identified studies met the inclusion criteria for oral corticosteroid therapy of NP ${ }^{(18)}$. Since 2007, only 3 randomized studies have reported the beneficial effect of combining oral and intranasal corticosteroid treatment ${ }^{(19-21)}$. The first study investigated patients treated with prednisone and intranasal budesonide ${ }^{(19)}$. The second study used only prednisolone compared to placebo ${ }^{(20)}$. The third study compared only methylprednisolone compared to placebo (21). Improvement in sense of smell with oral corticosteroid treatment was reported only in two of those studies ${ }^{(19,20)}$, although this effect was not sustained too long after discontinuing oral corticosteroids ${ }^{(20)}$. In a more recent study comparing the effect of oral prednisolone and placebo, both groups followed treatment with intranasal fluticasone propionate. The authors showed that initial oral corticosteroid therapy was effective on improving olfaction while this effect was less important when oral corticosteroids were discontinued and NP patients received only intranasal corticosteroid therapy ${ }^{(22)}$. The impact of NP was investigated and it has been demonstrated that nasal polyps impair QOL in all SF-36 domains namely that intranasal steroids improved the symptoms and the QOL in patients with NP ${ }^{(23)}$. Intranasal steroids are more effective than placebo in polyp size reduction ${ }^{(24)}$. A study revealed that a short course of oral steroids caused a significant improvement in all domains of SF-36. Long-term treatment with intranasal steroids maintained this improvement at the level of the Spanish general population. Although the reduction of polyp size is not really important, the clinical improvement especially the mental health is more significant ${ }^{(25)}$. Our study reveals that $\mathrm{QOL}$ in patients with NP is impaired where the physical health is being more impaired than the mental health and short course of oral steroids caused a significant improvement in all domains of RSDI and long-term treatment with intranasal steroids maintained this improvement. Over a dozen placebo controlled studies were published but the therapeutic effect was mostly studied for periods restricted to a few weeks ${ }^{(7,24,26)}$. Topical therapy has a definite beneficial effect on the clinical disorders and, on the polyp size but shows little activity on the sense of smell dysfunction. A study of 20 patients with CRS and nasal polyps treated for minimum 3 months with clarithromycin 400 mg/d ${ }^{(27)}$. The IL-8 levels were significantly higher before macrolide treatment than in the group whose polyps showed no change ${ }^{(28)}$. A study reported greater impairment of QOL when NP was associated with asthma showing that physical and mental functioning and vitality scores were significantly lower in asthmatic than in a non-asthmatic patient ${ }^{233}$. Further study demonstrated that asthma had an adverse impact on vitality and general health compared with rhinosinusitis alone, but no significant differences in postoperative SF-36 scores from patients with rhinosinusitis alone were established ${ }^{(29)}$.

This prospective study revealed that asthmatic patients had a higher nasal symptom score and worse QOL for physical, mental and social functioning than non-asthmatic patients. However, asthmatic and non-asthmatic patients had similar nasal symptoms, polyp size, and scored similar QOL domains after oral steroids, and these effects were maintained by long-term intranasal steroid therapy. The sense of smell worsened more at 12 months in non-asthmatic than in asthmatic patients with NP. In this study, a short course of oral steroids resulted in a significant reduction of polyp size in asthmatics, which was maintained on long-term intranasal steroid. It has been demonstrated that 
aspirin sensitivity has no further negative impact on QOL and no significant differences in nasal symptoms, polyp size, and QOL between aspirin-tolerant and aspirin-sensitive asthmatics prior and after short course of oral steroids or on long-term intranasal steroid treatment ${ }^{(30)}$. In our study, there was no significant difference in polyp size score between right and left nasal cavity in aspirin tolerant patients prior and after a short course of oral prednisolone and long-term intranasal steroids. On the contrary, there was significant difference noted in polyp size between right and left nasal cavities in aspirin intolerant patients. There has been a lack of correlation between endoscopy scores and self-related symptom scores or RSDI total scores in a nonsurgical population of 53 patients with CRS but without regard for polyp status ${ }^{(31)}$.

\section{Conclusion}

The improvements in QOL may be related to a patient's perception of changes in nasal obstruction, improved ventilation and olfaction, and reduced facial pressure and positive correlation between changes in endoscopy score and QOL subscale score, indicating that optimal medical therapy has dual effect on subjective and objective outcome in NP patients.

\section{Acknowledgement}

The authors thank all the staff of the Otorhinolaryngology clinic at Universiti Kebangsaan Malaysia Medical Centre for their contribution to this work in one way or another. The authors also thank Dr James Walsh from United Kingdom for his kind support in terms of grammar correction and scientific advice.

\section{Authorship contribution}

$\mathrm{SKH}$ : Principal investigator and corresponding author. SH: Co supervisor. BWS: Supervisor

\section{Conflicts of Interest}

No conflict of interest exists.

\section{References}

1. Fokkens WJ, Lund VJ, Mullol J, et al. EPOS2012, European position paper on rhinosinusitis and nasal polyps. Rhinology Suppl 2012; 23: 1-136.

2. Shahizon AMM, Suraya A, Rozman Z, Aini AA, Gendeh BS. Correlation of computed tomography and nasal endoscopic findings in chronic rhinosinusitis. Med J. Malaysia 2008; 63: 211-215.

3. Hedman J, Kaprio J, Poussa T. Prevalence of asthma, aspirin intolerance, nasal polyposis and COPD in population based study. In J Epidemiol 1999; 28: 717-772.

4. Kim JW, Hong SL, Kim YK. Histological and immunological features of non-eosinophilic nasal polyps. Otolaryngol Head Neck Surg 2007; 137: 925-930.

5. Soler ZM, Smith TL. Quality of life outcomes after functional endoscopic sinus surgery. Otolaryngol Clin N Am. 2010; 43: 605-612.

6. Lildholdt T, Mygind N. Effect of corticosteroids on nasal polyps: evidence from controlled trials. In nasal polyposis: an inflammatory disease and its treatment. Edited by Mygind N, LildholdtT.Copenhagen: Munksgaard: 1997: 160-169.

7. Lidholdt $\mathrm{T}$, Rundcrantz $\mathrm{H}$, lindqvist $\mathrm{N}$ Efficacy of topical corticosteroids powder for nasal polyp. A double-blind, placebocontrolled study of budesonide. Clinical otolaryngology 1995; 20: 26-3028.

8. Katsuta S, Osafune H, Takita R. [Therapeutic effect of roxithromycin on chronic sinusitis with nasal -- polyps clinical, computed tomography, and electron microscopy analysis]. Nihon Jibiinkoka Gakkai Kaiho 2002; 105: 1189-1197.

9. Stjarne P, Mosges $R$, Jorissen $M$ Randomized controlled trial of mometasone furoate nasal spray for the treatment of nasal polyposis. Arch Otolaryngol Head Neck Surg. 2006; 132: 179-185.

10. Ichimura, K, Shimazaki, Y, Ishibashi, T. Effect of new macrolide roxithromycin upon nasal polyps associated with chronic sinusitis. Auris Nasus Larynx. 1996; 23: 48-56.

11. Lildholdt T, Rundcrantz H, Bende M, Larsen K. Glucocorticoid treatment for nasal polypos. The use of topical bedsonide powder, intramuscular betamethasone and surgical treatment. Arch Otolaryngol Head Neck Surg. 1997; 123: 595-600

12. Fokkens WJ, Lund VJ, Mullol J, Bachert C Alobid I, Baroody F, et al. EPOS 2012: European position paper on rhinosinusitis and nasal polyps 2012. A summary for otorhinolaryngologists. Rhinology. 2012; 50: 1-12.

13. Johansen LV, Illum P, Kristensen S, Winter L, Petersen SV, Synnerstad B. The effect of rhinocort in the treatment of small and medium sized nasal polyps. Clin Otolaryngol. 1993; 18: 524-527.

14. Larsen K, Tos M. The estimated incidence of symptomatic nasal polyps. Acta Otolaryngol. 2002; 122: 179-182.

15. Van Camp P, Clement PAR. Results of oral steroid treatment in nasal polyposis. Rhinology. 1994; 32: 5-9.

16. Holmberg K, Juliusson S, Balder B, Smith DL, Richards DH, Karlsson G. Fluticasone propionate aqueous nasal spray in the treatment of nasal polyposis. Ann Allergy Asthma Immunol 1997; 78: 270-276.

17. Nores JM, Avan P, Bonfils P. Medical management of nasal polyposis: a study in a series of 152 consecutive patients. Rhinology 2003; 41: 97-102.

18. Patiar S, Reece P. Oral steroids for nasal polyps. Cochrane Database Syst Rev 2007; 1 : CD005232
19. Benitez P, Alobid I, de Haro J, Berenguer J , Bernal-Sprekelsen M, Pujols L, et al. A short course of oral prednisone followed by intranasal budesonide is an effective treatment of severe nasal polyps. Laryngoscope. 2006; 116: 770-775.

20. Hissaria P, Smith W, Wormald PJ, Taylor J, Vadas M, Gillis D, et al. Short course of systemic corticosteroids in sinonasal polyposis: a double-blind, randomized, placebocontrolled trial with evaluation of outcome measures. J Allergy Clin Immunol. 2006: 118: 128-133.

21. Van Zele T, Gevaert P, Holtappels G, et al. Oral steroids and doxycycline: two different approaches to treat nasal polyps. J Allergy Clin Immunol. 2010; 125: 1069-1076.e4.

22. Vaidyanathan S, Barnes M, Williamson $P$, Hopkinson P, Donnan PT, Lipworth B. Treatment of chronic rhinosinusitis with nasal polyposis with oral steroids followed by topical steroids: a randomized trial. Ann Intern Med. 2011; 154: 293-302.

23. Jankowski R, Bodino C. Evolution of symptoms associated to nasal polyposis following oral steroid treatment and nasalization of the ethmoid-radical ethmoidectomy is functional surgery for NPS. Rhinology. 2003; 41: 211-219.

24. Radenne F, Lamblin C, Vandezande LM, Tille-Leblond I, Darras J, Tonnel AB, et al. Quality of life in nasal polyposis. J Allergy Clin Immunol. 1999; 104: 79-84.

25. Alobid I, Benitez P, Pujols L, Maldonado M, Bernal-Sprekelsen M, Morello A, et al. Severe nasal polyposis and its impact on quality of life. The effect of a short course of oral steroids followed by long-term intranasal steroid treatment. Rhinology. 2006; 44: 8-13.

26. Blomqvist EH, Lundblad L, Anggärd A, Haraldsson PO, Stjärne P. A randomized 
controlled study evaluating medical treatment versus surgical treatment in addition to medical treatment of nasal polyposis. Allergy Clin Immunol. 2001; 107: 224-228.

27. Mygind N, Lund VJ. Topical corticosteroid therapy in rhinitis. Clinical Immunother 1996; 5: 122-136.

28. Yamada T, Fujieda S, Mori S, Yamamoto $H_{\text {, }}$ Saito H. Macrolide treatment decreased the size of nasal polyps and IL-8 levels in nasal lavage. Am J Rhinol. 2000; 14: 143-148.

29. Meltzer EO, Charous BL, Busse WW, Zinreich J, Lorber RR, Danzig MR. Added relief in the treatment of acute recurrent sinusitis with adjunctive mometasone furoate nasal spray. J Allergy Clin Immunol. 2000; 106: 630-637.

30. Winstead W, Barnett S. Impact of endoscop- ic sinus surgery on global health perception: an outcomes study. Otolaryngol Head Neck Surg. 1998; 119: 486-491.

31. Alobid I, Benítez P, Bernal-Sprekelsen M. Nasal polyposis and its impact on quality of life. Comparison between the effects of medical and surgical treatments. Allergy 2005; 60: 452-458.
Dr Saleh Khaled Aboud

Department of Otorhinolaryngology

- Head and Neck Surgery

Malaysian Allied Health Sciences

Academy (MAHSA) University

Jalan Elmu off Jalan Universiti

59100 Kuala Lumpur

Malaysia

Tel: +603 $79652555,+60163566491$

Fax: +60379317118

E-mail: salehkh70@gmail.com 